\title{
Familial Aggregation of Amyotrophic Lateral Sclerosis
}

\author{
Fang Fang, $\mathbf{M D}^{1,2}$, Freya Kamel, $\mathrm{PhD}^{2}$, Paul Lichtenstein, $\mathrm{PhD}^{1}$, Rino Bellocco, $\mathrm{ScD}^{1,3}$, Pär \\ Sparén, $\mathbf{P h D}^{1}$, Dale $\mathbf{P}$ Sandler, $\mathbf{P h D}^{2}$, and Weimin Ye, MD, $\mathbf{P h D}^{1}$ \\ 1 Department of Medical Epidemiology and Biostatistics, Karolinska Institutet, Stockholm, Sweden \\ 2 National Institute of Environmental Health Sciences, National Institutes of Health, Department of \\ Health and Human Services, Research Triangle Park, NC
}

${ }^{3}$ Department of Statistics, University of Milano-Bicocca, Milan, Italy

\begin{abstract}
Objective-To assess the relative risk of amyotrophic lateral sclerosis (ALS) in families of ALS patients.

Methods-We conducted a cohort study based on the Swedish Multi-Generation Register (MGR) in 1961-2005. Among 6,671 probands (first ALS case in the family), 1,909 full siblings, 13,947 children, and 5,405 spouses were identified (exposed group). Other persons in MGR, who were siblings, children, or spouses to persons without ALS, served as the reference group.

Relative risks of ALS among the exposed group, compared to the reference group, were calculated from Poisson regression models. Concurrence of ALS within twins was assessed in 86,441 twin pairs registered in the Swedish Twin Register.
\end{abstract}

Results-Nine cases of ALS were noted among the siblings and 37 cases among the children of the probands, giving a 17-fold risk among the siblings (95\% confidence interval [CI], 8.1-30.4) and a 9-fold risk among the children (95\% CI, 6.2-12.0), compared to the reference group. Siblings and children had a higher excess risk if the proband was diagnosed at younger age, and the excess risks decreased with increasing age at diagnosis of the proband $(p<0.001)$. Spouses had no significantly increased risk $(p=0.27)$. Two cases were identified among the co-twins of ALS probands, giving a relative risk of 32 (95\% CI, 5.2-102.6).

Interpretation-The siblings and children of ALS patients have an around 10-fold risk of ALS compared to the reference group. The excess risks vary with both age and kinship, indicating a major genetic role in familial ALS.

\section{Introduction}

Amyotrophic lateral sclerosis (ALS) is a neurodegenerative disease affecting motor neuron function. Although most ALS cases occur sporadically, up to ten percent of patients have a familial form.1 Mutations in several genes have been identified in familial ALS, including $S O D 1$, which is responsible for about $20 \%$ of the familial cases in an autosomal dominant form. Five other dominant loci - $A L S 3, A L S 4, A L S 6, A L S 7$, and $A L S 8$, as well as two recessive loci - $A L S 2$ and $A L S 5$ have also been suggested to play a role in familial ALS.2 More recently, the focus of research has moved from attempting to identify the "simple" genetics of a clearly Mendelian disorder to the "complex" genetics of the sporadic disease,

Correspondence to: Fang Fang.

Correspondence to: Fang Fang Department of Medical Epidemiology and Biostatistics Karolinska Institutet PO Box 28117177 Stockholm, Sweden Email: fang.fang @ki.se Tel: +46 852486113 Fax: +46 8314975. 
which may involve multiple genes. 3 Other unidentified or unconfirmed genes together with other non-genetic factors may also have impact on ALS etiology.

The magnitude of relative risk for ALS in families of ALS patients, compared to other families, has seldom been assessed.4-6 The most accurate way of establishing this relative risk is to follow the families of ALS patients prospectively. The availability of several nationwide registers in Sweden, including the Swedish Inpatient Register, the Causes of Death Register, and the Multi-Generation Register, provided us a unique opportunity to assess the relative risks of ALS among the siblings, children, and the spouses of ALS patients, compared to a reference population who had relatives without ALS. The additional impact of age and kinship on the relative risks were also assessed.

\section{Methods}

ALS cases

First, we identified all discharge records from the Swedish Inpatient Register between 1964 and 2005 with ALS as either the main or a secondary diagnosis. The Inpatient Register was established in 1964/1965 by the Swedish National Board of Health and Welfare, and has achieved nationwide coverage since 1987.7 Second, we identified all death records with ALS as the underlying cause of death from the Causes of Death Register between 1961 and 2004. ALS was coded according to the International Classification of Diseases $7^{\text {th }}$ Version (ICD-7) "356,10" before 1969, ICD-8 “348,00" from 1969 through 1986, ICD-9 “335C" from 1987 through 1996, and ICD-10 "G12.2" from 1997 and onward. By pooling cases from these two registers, we identified a total of 9,457 individuals with ALS. Duplicated cases identified in both registers were excluded. Register data were linked via the national registration number, a unique personal identifier of all Swedish residents.

\section{Exposed group}

The Swedish Multi-Generation Register contains information on all residents in Sweden who were born in 1932 or later and alive in 1961 ("index persons") together with their parents. 8 Familial linkage, i.e., parental information, is available for $>95 \%$ of individuals who died before 1968, about 60\% for those died between 1968 and 1990, and $>90 \%$ for those alive in 1991. Individuals deceased before 1991 were deleted from the register by parish civil registration offices, which were responsible for local population registration at that time. Among these deleted individuals, almost 100\% of deaths before 1968 and about 60\% of deaths between 1968 and 1990 could be re-identified from other sources, i.e., personal records and Statistics Sweden's register of births, and are included in the Register. Since 1991, the tax offices have been responsible for the local population registration and have supplied complete data to the Register.8

The first ALS case in each family was defined as the proband. The "exposed group" was restricted to the full siblings that were born as singletons (termed "singleton full siblings"), and children of the probands. Because siblings were identified through their parents, we could identify siblings for only 20\% of the ALS cases who were born since 1932 and had identifiable parents. We also assessed the relative risk of ALS among the spouses as the non-blood-related relatives of ALS probands. In this Register, spouses could be identified only through a common biological child, so we defined spouses as those sharing at least one identifiable biological child with the probands, whether married or not.

For 6,671 (70\%) of the 9,457 ALS cases, we identified 2,051 siblings, 14,615 children, and 6,851 spouses in total. Through cross-linkages to the Inpatient Register, Causes of Death Register, and Migration Register, the relatives were followed from the diagnosis date of the probands or their own birth dates, whichever came later, to their own ALS diagnosis, death, 
emigration out of Sweden, or the end of follow-up (December $\left.31^{\text {st }}, 2005\right)$, whichever occurred first. We excluded 2,256 relatives that had emigrated or died before ALS diagnosis of the probands, leaving 1,909 siblings, 13,947 children, and 5,405 spouses in the final analysis. Person-years and ALS outcomes were first calculated among the siblings, children, and spouses separately. Later, analyses combining siblings and children were conducted to increase statistical power to investigate the relative risk of ALS among the first degree relatives.

\section{Reference group}

All individuals who were singleton full siblings, children, or spouses of another individual in the Multi-Generation Register, excluding those enrolled in the exposed group, served as the reference group. The Multi-Generation Register contains in total 13,605,121 individuals including 10,899,507 (80\%) as a sibling, child, or spouse of someone else. We excluded 21,261 individuals that were included in the exposed group and 75,274 with erroneous information discovered in register linkage, leaving 10,802,972 in the final reference group. Individuals in the reference group were followed from January $1^{\text {st }}, 1961$ (when ALS diagnosis was first available in the Causes of Death Register) or their own birth dates, whichever came later, to the date of ALS diagnosis, death, emigration out of Sweden, or end of follow-up, whichever occurred first.

\section{Twin analysis}

The Swedish Twin Register was first established in the late 1950s.9 It contains 172,890 twins including 86,441 twin pairs and eight twins without a corresponding co-twin. Zygosity information, as determined by questions on childhood resemblance, was also identified from this register. Self-reported zygosity has been validated with DNA markers in a subsample of 199 twin pairs and was proved correct in 99\% of the twin pairs.9 We linked the Twin Register to the Inpatient Register and Causes of Death Register to identify the first ALS cases in all twin pairs (probands). The co-twins of probands were considered the exposed group. All other twins composed the reference group. The exposed and reference groups were followed as described above for the Multi-Generation Register. Analyses were first performed among all twins and later stratified by zygosity to illustrate the relative risk difference between monozygotic and dizygotic twins.

\section{Statistical analysis}

We used log-linear Poisson regression models to calculate the overall relative risks (RRs) and 95 percent confidence intervals (CIs) for ALS, as the ratio of ALS incidence rates of the exposed group to that of the reference group. We adjusted for attained age at follow-up ( $₫ 44,45-54,55-64$, and $\ 65$ years), sex, and calendar period (1961-1975, 1976-1990, and 1991-2005) in all statistical models. The log-transformed person-years were used as the offset variable in the models. Pearson's $\chi^{2}$ test was used to check the goodness of fit of the models. The exposed group was then broken down by age at diagnosis of ALS probands ( $\leq 44,45-54,55-64,65-74$, and $\geq 75$ years) and kinship (siblings or children). We further conducted analyses stratified by sex among all relatives, and by attained age at follow-up ( $\leq 44,45-54,55-64$, and $\succ 65$ years), either among all relatives or among siblings and children separately. All $p$ values were two-sided and the results were considered as statistically significant at $p<0.05$. All the analyses were performed using SAS, version 9.1 (SAS Institute, Cary, NC, USA). The study was approved by the Regional Ethics Committee at Karolinska Institutet. 


\section{Results}

The characteristics of the probands together with their siblings, children, and spouses are shown in Table 1. The mean age at diagnosis for the probands was 66.1 years. The mean age at the end of follow-up among the siblings was 57.6 years, among the children 51.0 years, and among the spouses 75.4 years. We identified nine cases of ALS among the siblings, 37 cases among the children, and eight cases among the spouses during follow-up, with a mean age at ALS diagnosis of 57.3, 53.8 and 71.2 years, respectively.

In the analysis pooling siblings and children together, compared with the reference group, we found a 10-fold risk of ALS (RR, 9.7; 95\% CI, 7.2-12.8) among the first degree relatives of ALS probands (Table 2). The relative risk was highest when the proband was diagnosed at the youngest age ( $\leq 44$ years) and decreased with increasing age at diagnosis of the probands ( $p$ value for trend $<0.001$ ). Siblings had a substantially higher increased risk than children (RR 16.8 versus 8.8). Children with a maternal proband had also a higher relative risk than children with a paternal proband (RR 11.7 versus 6.5 ). Analysis stratified by sex of the first degree relatives did not show a great difference between men and women (Table 3). The absolute incidence rates of ALS increased along with increasing attained age at followup among both the exposed and reference groups (Figure). But a pattern of decreasing relative risks along with increasing attained age at follow-up was noted ( $p$ value for trend $<$ 0.0001) (Table 3 and Figure). The relative risks appeared higher among the siblings than among the children in most age groups, except at the group of 55-64 (data not shown).

Eight cases of ALS were identified among the spouses (4 husbands and 4 wives) rendering no statistically significant elevated risk of ALS compared to the reference group (RR, 1.5; 95\% CI, 0.7-2.8). The relative risks did not differ significantly between husbands and wives or by age at diagnosis of the probands (data not shown).

To address potential concerns of the validity of ALS diagnosis, two sensitivity analyses were performed. First, we restricted the analysis to cases identified from the Inpatient Register only; 32 cases were observed among the siblings and children (related to 5,291 probands), giving a RR of 9.2 (95\% CI, 6.4-12.8), and seven cases among the spouses, giving a RR of 1.7 (95\% CI, 0.7-3.4). Second, we identified another 296 probands with ALS as a contributory cause of death and their 41 siblings, 596 children, and 217 spouses. One additional child case was observed giving a RR of 8.6 among the children of the probands (95\% CI, 6.1-11.6).

In the twin analysis, we identified 82 cases of ALS from 5,907,828 person-years accumulated during follow-up in the reference group (crude incidence rate: 1.39/100,000 person-years). In the exposed group, two cases of ALS were observed out of 1,069 personyears (crude incidence rate: 187.1/100,000 person-year). The RR of ALS for the exposed group was 32.1 (95\% CI, 5.2-102.6). In the analysis stratified by zygosity, we found that both concurrent cases were monozygotic; the corresponding RR for monozygotic co-twins was 153 (95\% CI: 23.8-557.0).

\section{Discussion}

We observed an about 10-fold risk of ALS among the singleton full siblings and children of ALS patients, with a higher relative risk among the siblings. A few studies have assessed the association between a family history of ALS and the risk of ALS 4-6, with one study showing an around three-fold 5 and another more than 10-fold 4 risk among families of ALS patients. The various findings are likely due to different study designs and small sample sizes in these studies. Similar to our study, the only twin study available to date found 2 out of 26 monozygotic, but none out of 51 dizygotic, twin pairs with concordant ALS.10 
Contrary to the siblings and children, no statistically significant excess risk of ALS was noted among the spouses of ALS patients in our study. Spouses identified via a common child might not in fact live together with the proband, thus could possibly lead to an underestimated relative risk if the shared adulthood environment does matter. Alternatively, genetic factors could clearly contribute to the different relative risks found between the blood relatives and the spouses. Other non-genetic factors specifically shared by the blood relatives, but not spouses, and the probands may have also contributed to the elevated risk. Retroviral infection, for instance, may be a potential candidate. A recent study showed that ALS cases and their blood relatives had similar loads of serum reverse transcriptase activity (an enzyme characterizing retroviral infections), while the loads were lower in spouses, who had levels similar to that of the unrelated controls.11

Although the absolute risks of ALS in siblings and children of ALS probands increased with age (Figure), as were true in the general population, the relative risks of ALS decreased with both increasing age of ALS diagnosis of the probands (Table 2) and increasing attained age of the relatives (Figure). The result is consistent with findings from other neurodegenerative disorders such as Alzheimer's disease, where the relative risk peaked among the younger relatives of younger probands and dropped sharply both as the age at onset of the proband and the attained age of the relative increased 12 and Parkinson's disease, where the relative risk among relatives of early-onset probands was 4.7, while among relatives of late-onset probands 2.7. 13

The siblings appeared to have a higher relative risk compared to the children of ALS patients. The difference persisted after multiple adjustments for attained age at follow-up, sex, and calendar period. One possible explanation is that siblings are on average older than children and thus more likely to develop an age-dependent disease like ALS. In our study, the mean age at the end of follow-up between siblings and children differed by seven years. Separate analyses for siblings and children showing higher relative risks for the siblings in most age groups allayed such a concern. Given that siblings and children share the same number of genes with the probands, other explanations should be sought. One candidate explanation is recessive gene action. Siblings have the same chance as the proband case to inherit both recessive alleles from their common parents and develop the phenotype, while children inherit only one allele from the proband parent and their phenotypes thus depend on the other parent. Another potential explanation is the same early life exposures shared by the proband and their siblings but not their children, such as childhood infections which may modify the risk of ALS later in life.

We have no clear explanation for the relative risk difference on having a maternal versus a paternal proband, noted in our study. It is possible that, in some cases, the father reported in the registry was not the biological father of the child. Hypotheses concerning mitochondrial inheritance, parent-of-origin, and epigenetic phenomenon could be of value for further investigation. Preferential maternal inheritance through mitochondrial DNA has been suggested playing a role in familial ALS with yet conclusive evidence.14,15 Parent-oforigin effect operating through the maternal lineage, as proposed for multiple sclerosis, 16 may also be possible.

Our study had potential limitations. First, we had around $40 \%$ missing data in the MultiGeneration Register for individuals deceased between 1968 and 1990. If some deceased ALS cases belong to this missing, their corresponding relatives would be classified as part of the reference group, leading to an elevated baseline risk and an underestimated relative risk. Second, we could only identify ALS cases from the registers and may have lost some cases neither hospitalized nor deceased. Death certificate is shown able to identify 70-90\% of ALS cases 17 and in our data, for example, $77 \%$ of cases first hospitalized in 1991 were also 
found in the Causes of Death Register. We thus believe that these two registers should have captured the vast majority of ALS cases in Sweden. Given the big sample size, we were not able to verify the accuracy of ALS diagnosis. However, our sensitivity analyses by excluding death-certificate only cases or including deaths with ALS as a contributory cause showed largely unchanged results. The third limitation is that register-based data had no information on other features of interest including site of disease onset, thus preventing evaluation of relative risk variation associated with these features.

Genetic factors may play a role in the so-called sporadic cases. The discovery of mutations in SOD1 in 2-7\% of sporadic cases 18-21 supports this notion. Unfortunately, we had no information on prior familial history of ALS for probands in our study. Under some assumptions, we can, however, roughly estimate the genetic effects on sporadic ALS cases. If we assume that number and age distribution of first degree relatives did not differ between familial and sporadic probands, the accumulated person-time can be partitioned into two parts, $10 \%$ for familial and $90 \%$ for sporadic probands, given that around $10 \%$ cases are familial. By multiplying the age-specific incidence rates of the reference group to the observed person-time, as well as an assumed "real" relative risk for familial ALS (contrary to the observed relative risk in our study), the expected number of ALS cases in relatives of familial probands could be calculated (termed "A"). Similarly, the expected number of cases among relatives of sporadic probands, assuming no genetic effects, can also be estimated (termed "B"). The role of genetic component in the $90 \%$ sporadic cases could thus be assessed as a ratio (((observed ALS cases) - A)/B). We observed 46 cases among the siblings and children, thus, by assuming a "real" relative risk for familial ALS as 75 (half the value of monozygotic co-twins), the ratio was 3.9 for siblings and children of sporadic probands.

Finally, we observed only two monozygotic twin pairs with concurrent ALS and could not compare the relative risks between monozygotic and dizygotic co-twins of ALS patients. But the fact that the relative risk among all co-twins of ALS probands was double that of the singleton siblings together with the even more striking relative risk among the monozygotic co-twins strongly suggests a significant role of genetics in ALS etiology. Studies able to pool twin registers from different countries are preferably warranted.

Despite limitations discussed above, our study has several significant strengths. First is the prospective cohort design, population-based approach, large sample size, and the close to complete and long-term follow-up. Second is the unbiased identification of familial history of ALS through computerized nationwide registers, which minimized the possibility of exposure misclassification. And finally, we were able to compare the relative risks of ALS between blood relative and spouses, between siblings and children, and between co-twins and singleton full siblings, of ALS patients. These strengths ensured both the validity and uniqueness of our study.

In conclusion, we observed an almost 10-fold increase in ALS risk among the siblings and children, but not spouses, of ALS patients in Sweden. The excess risk appeared higher among the siblings compared to the children, and probably also among those with a maternal proband compared to those with a paternal proband. The excess risks were most notable when the proband patients were diagnosed at a younger age, indicating a major genetic role in familial ALS, particularly for early-onset ones. Future studies in different populations are warranted to confirm our observations.

\section{Acknowledgments}

This work was partly funded by Björklunds Fond and by intramural funding from the National Institutes of Health, National Institute of Environmental Health Sciences. 


\section{References}

1. Rowland LP, Shneider NA. Amyotrophic lateral sclerosis. N Engl J Med. 2001; 344:1688-1700. [PubMed: 11386269]

2. Pasinelli P, Brown RH. Molecular biology of amyotrophic lateral sclerosis: insights from genetics. Nat Rev Neurosci. 2006; 7:710-723. [PubMed: 16924260]

3. Orrell RW. Understanding the causes of amyotrophic lateral sclerosis. N Engl J Med. 2007; 357:822-823. [PubMed: 17671249]

4. Kamel F, Umbach DM, Munsat TL, et al. Association of cigarette smoking with amyotrophic lateral sclerosis. Neuroepidemiology. 1999; 18:194-202. [PubMed: 10364720]

5. Cruz DC, Nelson LM, McGuire V, Longstreth WT Jr. Physical trauma and family history of neurodegenerative diseases in amyotrophic lateral sclerosis: a population-based case-control study. Neuroepidemiology. 1999; 18:101-110. [PubMed: 10023133]

6. Majoor-Krakauer D, Ottman R, Johnson WG, Rowland LP. Familial aggregation of amyotrophic lateral sclerosis, dementia, and Parkinson's disease: evidence of shared genetic susceptibility. Neurology. 1994; 44:1872-1877. [PubMed: 7936240]

7. Nyren O, McLaughlin JK, Gridley G, et al. Cancer risk after hip replacement with metal implants: a population-based cohort study in Sweden. J Natl Cancer Inst. 1995; 87:28-33. [PubMed: 7666459]

8. Statistics Sweden. Multi-generation register 2005 A description of contents and quality. Statistics Sweden; Örebro: 2005.

9. Lichtenstein P, De Faire U, Floderus B, et al. The Swedish Twin Registry: a unique resource for clinical, epidemiological and genetic studies. J Intern Med. 2002; 252:184-205. [PubMed: 12270000]

10. Graham AJ, Macdonald AM, Hawkes CH. British motor neuron disease twin study. J Neurol Neurosurg Psychiatry. 1997; 62:562-569. [PubMed: 9219739]

11. Steele AJ, Al-Chalabi A, Ferrante K, et al. Detection of serum reverse transcriptase activity in patients with ALS and unaffected blood relatives. Neurology. 2005; 64:454-458. [PubMed: 15699374]

12. Silverman JM, Ciresi G, Smith CJ, et al. Variability of familial risk of Alzheimer disease across the late life span. Arch Gen Psychiatry. 2005; 62:565-573. [PubMed: 15867110]

13. Thacker EL, Ascherio A. Familial aggregation of Parkinson's disease: a metaanalysis. Mov Disord. 2008; 23:1174-1183. [PubMed: 18442112]

14. Orrell RW, Schapira AH. Mitochondria and amyotrophic lateral sclerosis. Int Rev Neurobiol. 2002; 53:411-426. [PubMed: 12512348]

15. Bradley M, Bradley L, de Belleroche J, Orrell RW. Patterns of inheritance in familial ALS. Neurology. 2005; 64:1628-1631. [PubMed: 15883330]

16. Ebers GC, Sadovnick AD, Dyment DA, et al. Parent-of-origin effect in multiple sclerosis: observations in half-siblings. Lancet. 2004; 363:1773-1774. [PubMed: 15172777]

17. Chio A, Magnani C, Oddenino E, et al. Accuracy of death certificate diagnosis of amyotrophic lateral sclerosis. J Epidemiol Community Health. 1992; 46:517-518. [PubMed: 1479322]

18. Andersen PM, Nilsson P, Ala-Hurula V, et al. Amyotrophic lateral sclerosis associated with homozygosity for an Asp90Ala mutation in CuZn-superoxide dismutase. Nat. Genet. 1995; 10:6166. [PubMed: 7647793]

19. Jackson M, Al-Chalabi A, Enayat ZE, et al. Copper/zinc superoxide dismutase 1 and sporadic amyotrophic lateral sclerosis: analysis of 155 cases and identification of a novel insertion mutation. Ann Neurol. 1997; 42:803-807. [PubMed: 9392581]

20. Jones CT, Shaw PJ, Chari G, Brock DJ. Identification of a novel exon 4 SOD1 mutation in a sporadic amyotrophic lateral sclerosis patients. Mol. Cell. Probes. 1994; 8:329-330. [PubMed: 7870076]

21. Jones CT, Swingler RJ, Brock DJ. Identification of a novel SOD1 mutation in an apparently sporadic amyotrophic lateral sclerosis patient and the detection of Ile113Thr in three others. Hum Mol Genet. 1994; 3:649-650. [PubMed: 8069312] 


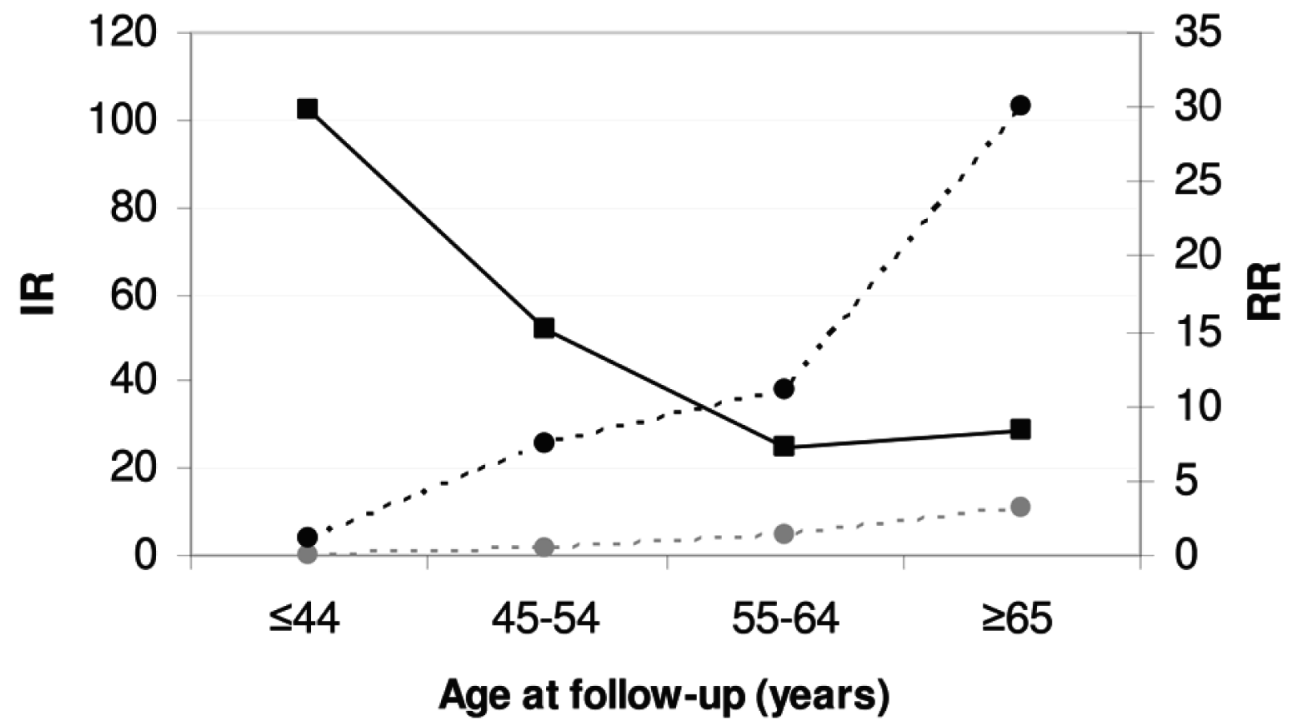

Fig.

Incidence rates (IR, per 100,000 person-years; among the first degree relatives of ALS probands and the reference group, respectively) and relative risks (RR; among the first degree relatives of ALS probands, compared to the reference group) of ALS, by attained age at follow-up. Gray dashed line with circles = IR Reference. Black dashed line with circles = IR Exposed. Solid black line with squares $=$ RR. 


\section{Table 1}

Characteristics of ALS proband cases, their full siblings, children, and spouses in Sweden, 1961-2005

\begin{tabular}{|c|c|c|c|}
\hline & Men & Women & Total \\
\hline \multicolumn{4}{|l|}{ Proband cases } \\
\hline No. of proband cases & 3,843 & 2,828 & 6,671 \\
\hline Mean age at diagnosis (years) & 65.5 & 67.0 & 66.1 \\
\hline \multicolumn{4}{|l|}{ Siblings } \\
\hline No. of siblings & 987 & 922 & 1,909 \\
\hline Mean follow-up duration (years) & 7.4 & 6.8 & 7.1 \\
\hline Mean attained age at the end of follow-up (years) & 57.5 & 57.8 & 57.6 \\
\hline No. of ALS cases among siblings during follow-up & 4 & 5 & 9 \\
\hline Mean age of siblings at diagnosis of ALS (years) & 58.0 & 56.8 & 57.3 \\
\hline \multicolumn{4}{|l|}{ Children } \\
\hline No. of children & 7,176 & 6,771 & 13,947 \\
\hline Mean follow-up duration (years) & 15.3 & 15.7 & 15.5 \\
\hline Mean attained age at the end of follow-up (years) & 50.8 & 51.3 & 51.0 \\
\hline No. of ALS cases among children during follow-up & 22 & 15 & 37 \\
\hline Mean age of children at diagnosis of ALS (years) & 53.8 & 53.7 & 53.8 \\
\hline \multicolumn{4}{|l|}{ Spouses } \\
\hline No. of spouses & 1,854 & 3,551 & 5,405 \\
\hline Mean follow-up duration (years) & 10.1 & 13.4 & 12.3 \\
\hline Mean attained age at the end of follow-up (years) & 76.6 & 74.7 & 75.4 \\
\hline No. of ALS cases among spouses during follow-up & 4 & 4 & 8 \\
\hline Mean age of spouses at diagnosis of ALS (years) & 71.2 & 71.2 & 71.2 \\
\hline
\end{tabular}




\section{Table 2}

Relative risk of ALS in full siblings and children of proband ALS cases, compared to risk of ALS in a reference population, Sweden, 1961-2005

\begin{tabular}{|c|c|c|c|c|}
\hline & Cases & Person-years & $\mathbf{R R}^{*}$ & $95 \% \mathrm{CI}^{\dagger}$ \\
\hline Reference group & 6,646 & $341,171,009$ & 1.0 & \\
\hline Exposed group & 46 & 229,638 & 9.7 & $7.2-12.8$ \\
\hline \multicolumn{5}{|c|}{ Age of proband at diagnosis of ALS } \\
\hline$\$ 44$ years & 3 & 14,784 & 36.5 & $9.1-94.7$ \\
\hline $45-54$ years & 6 & 32,835 & 16.0 & 6.4-32.4 \\
\hline $55-64$ years & 24 & 67,127 & 21.3 & 13.9-31.1 \\
\hline $65-74$ years & 10 & 77,713 & 5.6 & $2.8-9.8$ \\
\hline$\geq 75$ years & 3 & 37,179 & 2.2 & $0.6-5.7$ \\
\hline \multicolumn{5}{|c|}{ Kinship to the proband } \\
\hline Siblings & 9 & 13,540 & 16.8 & $8.1-30.4$ \\
\hline Children & 37 & 216,098 & 8.8 & $6.2-12.0$ \\
\hline Paternal proband & 15 & 130,202 & 6.5 & $3.7-10.3$ \\
\hline Maternal proband & 22 & 85,896 & 11.7 & 7.4-17.3 \\
\hline
\end{tabular}




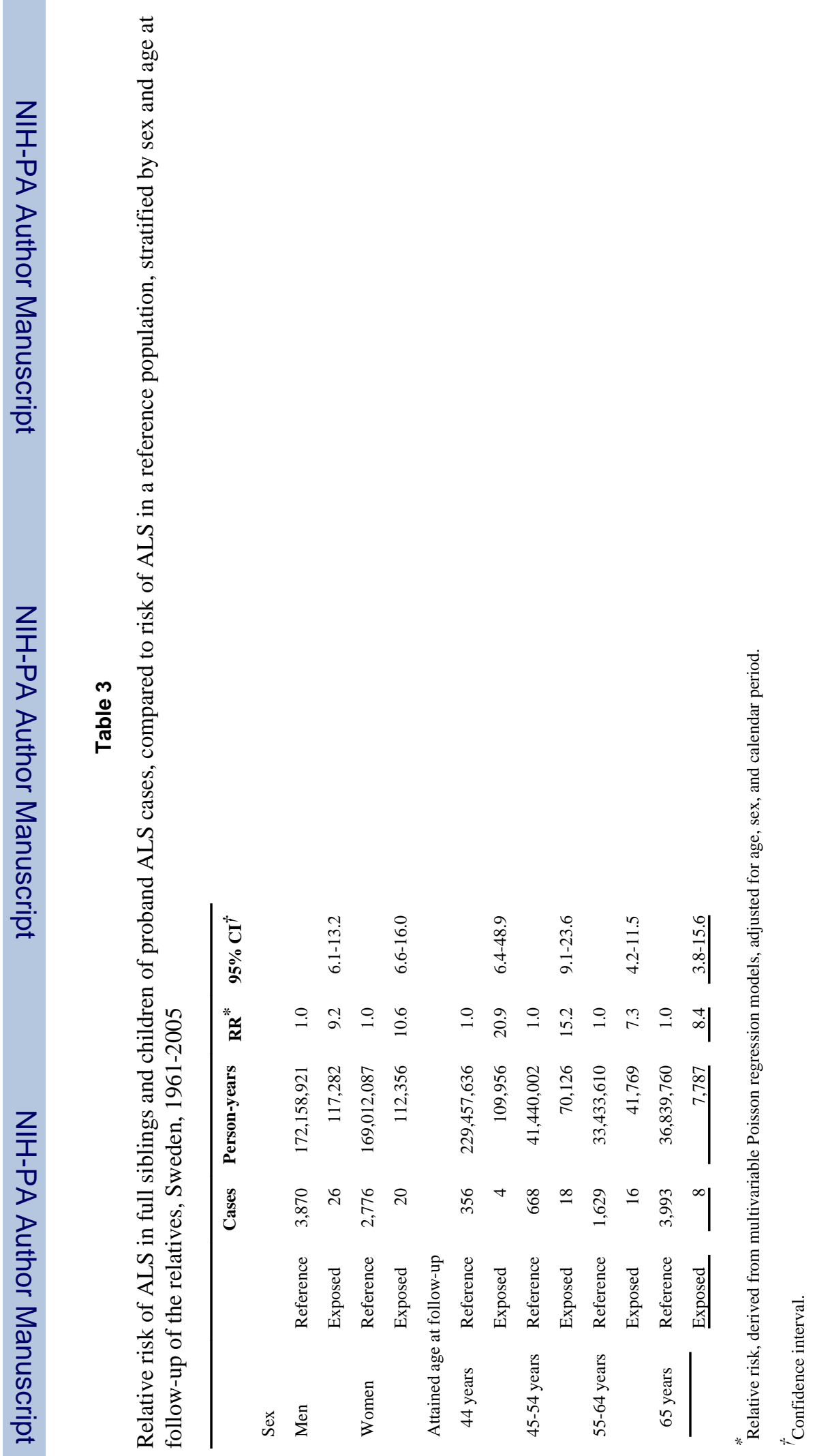

